\title{
The neurobiology of migraine
}

\author{
ANDREW CHARLES ${ }^{*}$ and K.C. BRENNAN \\ Headache Research and Treatment Program, Department of Neurology, David Geffen School of \\ Medicine at UCLA, Los Angeles, CA, USA
}

\section{ANATOMICAL COMPONENTS OF MIGRAINE}

\section{Cerebral cortex}

There is substantial clinical and imaging evidence for changes in cortical activity associated with migraine. Migraine is associated with a variety of symptoms that can be attributed to changes in cortical function. The most prominent among these are the visual changes associated with migraine aura that arise from altered function in the occipital lobe. Migraine patients may also experience cortical sensory, motor, language, or other cognitive dysfunction. These symptoms are typically described in the context of migraine with aura, but they may also occur with migraine that does not meet diagnostic criteria for migraine with aura. Functional imaging studies confirm that migraine is associated with dramatic changes in blood flow and metabolic activity in the cortex (Cutrer and Black, 2006). These imaging phenomena have been demonstrated primarily in patients with migraine with aura, but may also occur in patients with migraine without aura (Woods et al., 1994; Geraud et al., 2005). Migraine-related changes in blood flow and functional magnetic resonance imaging (fMRI) signal in the cortex are propagated with temporal and spatial characteristics that are remarkably similar to those of cortical spreading depression (CSD), the spreading wave of depolarization followed by suppression of electrocortical activity originally described by Leão in 1944 (Woods et al., 1994; Hadjikhani et al., 2001). The correlation between the characteristics of the clinical symptoms of migraine aura, CSD in animal models, and functional imaging has provided support for the long-standing hypothesis that CSD is a fundamental mechanism of migraine aura. A much more controversial question continues to be whether similar cortical phenomena may also occur in migraine without aura. As discussed below, distinct patterns of signaling in individual cellular compartments could underlie cortical activity that does not necessarily evoke classical aura symptoms.

Other evidence for fundamental changes in cortical excitability in migraine comes from clinical electrophysiology studies. A significant number of studies find an increased amplitude as well as a decreased habituation of cortical evoked potentials in migraine patients compared with controls during the interictal period, with normalization of these differences during the ictal period (Schoenen, 2006). Other studies also show that the threshold for generation of phosphenes by transcranial magnetic stimulation is reduced in

*Correspondence to: Andrew Charles MD, Headache Research and Treatment Program, Department of Neurology, David Geffen School of Medicine at UCLA, 635 Charles Young Drive, Los Angeles, CA 90095, USA, Tel: 310-206-7226, Fax: 310-206-6906, acharles@ucla.edu. 
migraine patients (Aurora et al., 1999, 2003; Gerwig et al., 2005). Migraine patients have also been reported to show reduced magnetic stimulation-induced suppression of visual accuracy (Aurora et al., 2007). These findings are consistent with an increased cortical excitability (or decreased inhibition) in patients with migraine.

However, there are also a significant number of studies that show either no differences in clinical electro-physiological parameters between migraine patients and controls, or in fact changes in the opposite direction consistent with a reduced cortical excitability in migraine patients (Ambrosini and Shoenen, 2006). The discrepancies between these studies may arise in part from methodological differences in the way the studies were performed. But another key explanation for these discrepancies is that the level of cortical excitability in migraine patients may vary substantially over time. Consistent with this idea, the thresholds for phosphene generation evoked by consecutive transcranial magnetic stimulation were found to be more variable in migraine patients than in controls (Antal et al., 2006). This suggests that, rather than having cortical excitability that can be characterized simply as either increased or reduced, migraine patients have a dysregulation of cortical excitability (Ambrosini and Shoenen, 2006; Stankewitz and May, 2007). Abnormally wide swings in cortical excitability are an appealing explanation for the complex variety of symptoms that are experienced by migraine patients.

\section{Brainstem}

There is also strong evidence that brainstem mechanisms play a significant role in the pathophysiology of migraine. Nausea, vertigo, and autonomic symptoms are among the clinical features of migraine that may arise from an alteration of signaling in the brainstem. It has also been suggested that the pain of migraine may arise primarily from the brainstem (Weiller et al., 1995; Tajti et al., 2001). Functional imaging studies of migraine patients consistently demonstrate activation of the brainstem during migraine attacks, particularly the region of the dorsolateral pons (Bahra et al., 2001; Afridi et al., 2005; Denuelle et al., 2007). Positron emission tomography (PET) and fMRI studies suggest that metabolism and function in the brainstem may also be chronically altered in patients with chronic migraine (Welch et al., 2001; Aurora et al., 2007). There have also been multiple reports of structural lesions in the brainstem that appear to cause headache in humans (Haas et al., 1993; Goadsby, 2002; Fragoso and Brooks, 2007). Furthermore, electrical stimulation in the region of the periaqueductal gray can evoke headache (Raskin et al., 1987).

\section{The trigeminal pathway}

Although it is clear from functional imaging studies that multiple brain regions involved in nociception are activated during a migraine attack (Weiller et al., 1995; Cao et al., 1999, 2002; Bahra et al., 2001; Afridi et al., 2005; Denuelle et al., 2007), the site where the initial activation of these nociceptive pathways occurs has not been determined with certainty. The idea that dilation of cerebral vessels is a primary cause of migraine pain has been challenged by a variety of evidence. However, the "trigeminovascular system" continues to be widely accepted as an important component of the headache. This concept developed based on the early observations that stimulation of trigeminally innervated vessels evoked pain in conscious patients, whereas stimulation of the brain parenchyma did not evoke discomfort 
(Penfield, 1935; Ray and Wolff, 1940). A role for the trigeminovascular system was supported by the observation that calcitonin gene-related peptide (CGRP), a neuropeptide known to be involved in cerebral vasoregulation, is increased in jugular venous blood during a migraine attack (Goadsby and Edvinsson, 1994). The idea of trigeminovascular activation has been reinforced by the effects of triptans, and more recently CGRP antagonists, whose effects have been presumed to be in large part mediated by inhibition of perivascular trigeminal nociceptive input. However, the findings that activation of 5-HT1 receptors by triptans at multiple other sites was involved in trigeminal nociception, including the nucleus caudalis, periaqueductal gray, and thalamus indicate that the perivascular trigeminal nerve endings are not necessarily the only site of action of these drugs (Goadsby and Hoskin, 1996; Ellrich et al., 2001; Boers et al., 2004; Shields and Goadsby, 2006). Other migraine therapies such as ergotamines, that had initially been presumed to act primarily at the peripheral trigeminal vascular complex, have also been shown to have central mechanisms of action (Hoskin et al., 1996).

It is also important to recognize that CGRP-containing neurons are found through the central and peripheral nervous system (including the trigeminal nucleus caudalis in the brainstem) (Tajti et al., 2001; Jenkins et al., 2004; Offenhauser et al., 2005; D'Hanis, 2007), and these neurons could represent an alternative source of the CGRP that is released during a migraine attack. Thus, although there is considerable indirect evidence for trigeminovascular activation in migraine, there is as yet no direct evidence that confirms this hypothesis. This may be because there is no reasonable way of measuring such activation in humans. However, it is interesting to note that trigeminal rhizotomy, even when complete disruption of trigeminal sensory function has been achieved, is not consistently effective in the prevention of migraine or cluster headache that is experienced on the side ipsilateral to the trigeminal deafferentation (Matharu and Goadsby, 2002). This observation suggests that it is possible for nociceptive pathways responsible for headache to be activated in the absence of peripheral trigeminal input from the same side on which the headache is experienced. Further investigation regarding the sites of actions of the triptans and CGRP receptor antagonists in humans may shed important light on this issue.

\section{Is there an anatomical sequence in migraine?}

The sequence of activation of different brain regions in migraine remains uncertain. At this stage it is not clear whether changes in cortical activity activate the brainstem, or vice versa. Alternatively, changes in these brain regions could occur in parallel, without an orderly sequence from one to the next. The typical occurrence of the migraine aura before migraine headache supports the hypothesis that cortical activation precedes brainstem activation. Consistent with this concept, important studies in experimental models demonstrate that it is possible for CSD to activate neurons in the trigeminal nucleus caudalis via trigeminal afferents (Bolay et al., 2002). Conversely, it has been shown that brainstem activation can evoke changes in cortical blood flow, raising the possibility that a process beginning in the brainstem could secondarily evoke some of the cortical phenomena of migraine (Adams et al., 1989; Goadsby and Duckworth, 1989). But it is also possible that both brain regions are activated simultaneously, a concept that is supported by the observations that, in some patients, clinical symptoms may occur without any clearly defined sequence that indicates 
alteration in the function of one region leading to change in function of another. Regardless of the order of their activation, however, it is clear that both cortical and brainstem signaling mechanisms are involved in migraine and are appealing targets for new therapeutic approaches for migraine.

\section{CELLULARCOMPONENTS OF MIGRAINE}

The concept of migraine as a primarily vascular headache has given way to the understanding that it is an episodic disorder of brain excitability that involves coordinated changes in neuronal, glial, and vascular function. Examination of the individual cellular components of migraine identifies new potential pathophysiological mechanisms, and new targets for treatment.

\section{Nociception}

It is generally assumed that migraine pain is initiated by activation of meningeal sensory afferents that synapse in the trigeminal nucleus caudalis, although, as discussed above, this assumption has not been definitively confirmed. Trigeminal neurons that innervate the meninges have properties that are in many ways similar to other visceral sensory neurons (Strassman and Levy, 2006). The only sensation that they appear to transmit is pain. However, the specific stimuli that trigger sensory neurons to produce migraine pain are not certain. A variety have been proposed, including mechanical pressure, changes in the ionic composition of the extracellular space (e.g., increased $\mathrm{K}^{+}$, increased osmolarity, and decreased $\mathrm{pH}$ ), neuropeptides (e.g., bradykinin, substance $\mathrm{P}$, endothelin, and CGRP), neurotransmitters (glutamate, serotonin, histamine, adenosine triphosphate, adenosine), eicosanoids, and nitric oxide. There may be subsets of trigeminal neurons that respond specifically to different stimuli with different thresholds. Individual neurons with conduction velocities consistent with $\mathrm{A}$ and $\mathrm{C}$ fibers have been identified, with different sensitivities to mechanical versus neurochemical stimuli, and different responses to repetitive or sequential combinations of stimuli (Levy and Strassman, 2002).

Peripheral trigeminal neurons, as with other neurons in the trigeminal pathway, show the phenomenon of sensitization, whereby exposure to an algesic stimulus lowers the threshold for the response of the neuron to the same stimulus, or a different stimulus (Strassman et al., 1996). For example, exposure to inflammatory mediators lowers the threshold of trigeminal neurons for responding to a mechanical stimulus (Levy and Strassman, 2002). This type of sensitization has been suggested as a mechanism whereby otherwise painless localized or diffuse changes in pressure (such as might occur with vascular pulsations) could result in the pulsating pain of migraine. It has been proposed that both peripheral and central sensitization are involved in the generation and maintenance of migraine pain, as well as the associated phenomenon of cutaneous allodynia.

\section{Cortical waves}

A complex interplay between neurons, glial cells, and vascular cells may be particularly critical in cortical mechanisms of migraine. As discussed above, the anatomical spread of migraine aura symptoms as well as the propagated changes in blood flow and metabolism 
observed in migraine patients has implicated the phenomenon of CSD in migraine. CSD was originally reported by Leão in 1944 as a suppression of electrical activity that spread slowly across large areas of the cortex (Leão, 1944). Subsequent recordings demonstrated that the suppression of activity was preceded by a dramatic depolarization, resulting in a "DC [direct current] shift" of the electrocortical signal, indicating that the CSD wave consists of a propagated wave of profound cortical activation followed by sustained inhibition of activity (Leão, 1947).

The clinical symptoms of migraine aura, as well as the clinical electrophysiological and transcranial magnetic stimulation responses of migraine patients, indicate a fundamental role for changes in neuronal excitability as a basis for increased cortical excitability in migraine. Other evidence comes from genetic and pharmacological studies. Mutations in neuronspecific genes have been identified as the cause of familial hemiplegic migraine in some families. These include mutations in the P/Q-type calcium channel (FHM1) (Ophoff et al., 1996; van den Maagdenberg et al., 2004), and SCN1A voltage-gated sodium channel (Dichgans et al., 2005). In each case, the resulting alteration of function of the channel results in an increase in the excitability of the neuron.

Pharmacological studies indicate that inhibition of neuron-specific receptors or channels can inhibit CSD. Inhibition of $\mathrm{N}$-methyl-d-aspartate (NMDA) receptors, which are expressed primarily on neurons, can inhibit the occurrence of CSD in vitro and in vivo preparations (Hernandez-Caceres et al., 1987; Peeters et al., 2007). CSD in vitro can also be blocked by inhibitors of P/Q-type calcium channels, which are specific to neurons (Kunkler and Kraig, 2004). Conversely, studies with a knock-in mouse model expressing a mutation of the P/Q calcium channel gene associated with familial hemiplegic migraine show that alteration in the function of this neuronal channel lowers the threshold for evoking CSD, and increases its rate of propagation (van den Maagdenberg et al., 2004). These studies indicate that neuronal hyperexcitability is involved in the triggering of CSD and its propagation.

\section{Astrocyte waves}

Glial cells may also play a key role in the changes in cortical activity associated with migraine. Direct evidence for such a role comes from the discovery that a mutation in an $\mathrm{Na}^{+} / \mathrm{K}^{+}$ATPase that is expressed primarily in astrocytes is responsible for familial hemiplegic migraine type 2 (De Fusco et al., 2003; Vanmolkot et al., 2006). In vitro studies indicate that this mutation reduces the function of the enzyme (Segall et al., 2005; Vanmolkot et al., 2006), an effect that would be expected to increase excitability by increasing extracellular $\mathrm{K}^{+}$. Astrocytes are abundant cells in the central nervous system that have been traditionally viewed as playing only a passive and supportive role in nervous system function. But recent studies demonstrate that astrocytes are capable of extensive intercellular signaling that can modulate both neuronal and vascular activity. Astrocytes express a variety of neurotransmitter receptors that allow them to respond to neuronal activity (Fellin et al., 2006). Conversely, they are capable of active release of transmitters, including glutamate and adenosine triphosphate (ATP), that can modulate neuronal function (Haydon and Carmignoto, 2006). Astrocytes are also in close contact with vascular cells via their endfeet, that enwrap blood vessels. Astrocyte signaling has been shown to modulate 
vascular tone directly, resulting in either vasoconstriction or vasodilation via release of eicosanoids, $\mathrm{K}^{+}$, and ATP (Zonta et al., 2003; Mulligan and MacVicar, 2004; Filosa et al., 2006; Takano et al., 2006).

Astrocytes are capable of extensive intercellular communication via increases in intracellular calcium concentration that are propagated from cell to cell in a wave-like pattern (Charles et al., 1991). The primary mechanism for these intercellular calcium waves appears to be release of ATP into the extracellular space and activation of purinergic receptors on adjacent cells (Guthrie et al., 1999). These intercellular calcium waves in astrocytes can be triggered by chemical, electrical, or mechanical stimuli, and spread with temporal and spatial characteristics that are remarkably similar to those of CSD (Charles, 1998). In fact, recent microscopic imaging studies have shown that calcium waves in astrocytes consistently occur in association with spreading depression, both in vitro and in vivo (Peters et al., 2003; Chuquet et al., 2007). However, each phenomenon can occur independently of the other. Inhibition of astrocyte calcium waves does not block spreading depression, and astrocyte calcium waves occur in the absence of spreading depression (Peters et al., 2003; Chuquet et al., 2007). Thus, they appear to be related phenomena that occur in parallel but without a requisite interdependence.

It has been assumed for decades that spreading depression as it is observed in animal models is the physiological basis for migraine aura. But the classical electroencephalogram (EEG) changes of CSD have not been observed in migraine patients. This could be because surface EEG recordings have not been sensitive enough to detect it. However, CSD in animal models is a profound neurophysiological event. It would be expected to produce not only EEG changes, but also a greater degree of neurological impairment than is observed in most migraine patients. A speculative alternative to classical CSD as a mechanism of the propagated cortical changes in migraine is a phenomenon that involves astrocyte waves. Astrocyte calcium waves are associated with release of significant amounts of ATP, glutamate, and vasoactive mediators (Haydon and Carmignoto, 2006). Based on this function, and based on their close spatial relationship with both neurons and vascular cells, astrocytes are ideally positioned to modulate widely propagated changes in both vascular activity and neuronal activity with the pattern that is observed in migraine patients. A primary role for astrocytes could explain both the propagated changes in blood flow and metabolism that are observed with functional imaging studies, as well as the cortical symptoms that in most patients are not as profound as might be expected to result from classical CSD. Even if astrocytes do not play such a primary role, it is likely that their activity represents a substantial component of the cellular pathophysiology of migraine.

\section{Vascular waves}

In addition to the changes in neuronal and glial cell function, migraine clearly involves significant changes in the activity of vascular cells. Mutations in genes encoding proteins expressed predominantly by vascular cells have been found in families with vasculopathies that have migraine as part of the clinical phenotype. Mutations in the gene encoding Notch3, which in adult humans is expressed predominantly in vascular smooth-muscle cells, cause cerebral autosomal-dominant arteriopathy with subcortical infarcts and leukoecephelopathy 
(CADASIL). This condition begins with migraine with aura in approximately one-third of patients (Dichgans et al., 1998). Mutations in the gene encoding the widely expressed exonuclease TREX1 also cause a vasculopathy that may have migraine as part of the phenotype (Richards et al., 2007).

As with astrocytes, it has long been assumed that vascular cells simply respond passively to changes in neuronal function. But it is important to keep in mind that vascular cells are also capable of active intercellular signaling, and can release messengers like nitric oxide that could potentially modulate the function of surrounding neurons and glia. As mentioned above, the longstanding hypothesis that migraine pain is a direct consequence of vasodilation has been increasingly challenged by functional imaging studies in migraine patients. The classic blood flow studies by Olesen and colleagues (1990) clearly demonstrated an increase in blood flow in patients with migraine. However, they found that the onset of pain preceded the onset of increased blood flow, and in fact began during the period of hypoperfusion that was associated with migraine aura. Conversely, the phase of increased blood flow often persisted for a significant duration after the pain of migraine had stopped. These studies suggest that vasodilation and the pain of migraine are not necessarily temporally correlated.

A variety of other functional imaging studies with PET and fMRI techniques also show cortical hypoperfusion during the onset of migraine pain (Woods et al., 1994; Hadjikhani et al., 2001; Geraud et al., 2005). Still other more recent studies with pharmacologically induced migraine also demonstrate that the onset of pain is not correlated with vasodilation. Olesen and colleagues found that, with migraine induced by sildenafil, the pain of migraine did not begin until after recovery from the drug-induced cerebral vasodilation, as indicated by transcranial Doppler (Kruuse et al., 2003). Similarly, Schoonman and colleagues (2008) used magnetic resonance angiography techniques to show that migraine pain evoked by nitroglycerin did not occur until after recovery from nitroglycerin-induced cerebral vasodilation.

The complex vascular changes that are observed with CSD may provide some insight into the complex vascular phenomena seen with functional imaging studies in migraine patients. Spreading depression in mice is associated with a multiphasic vascular response. There is an initial dilation of cortical surface vessels that may be actively propagated along the vessel via an intrinsic vascular mechanism (Brennan et al., 2007b). This is followed by a profound constriction of the vessels, after which there is subsequent vasodilation and then eventual recovery to normal caliber (Ayata et al., 2004; Brennan et al., 2007b). There may be significant species-specific and methodology-related differences in the presence and extent of the vasoconstriction component of the surface arteriolar response to CSD. In humans, a significant vasoconstriction is supported by functional imaging studies that consistently show a hypoperfusion associated with migraine aura, and even in migraine without aura (Woods et al., 1994; Cao et al., 1999; Geraud et al., 2005). In vivo imaging studies in rodent models provide evidence that astrocyte calcium waves mediate the propagated vasoconstriction associated with CSD, indicating a primary role for glial cells in this process (Chuquet et al., 2007) (Figure 7.1). 
Vasoconstriction could be involved in the generation of migraine pain via a variety of mechanisms. First, constriction is associated with the release of messengers and peptides that are believed to be involved in the generation of migraine. CGRP, widely accepted as a potentially important mediator of migraine pain, is released by perivascular neurons in response to vasoconstriction as part of a reflex arc whose purpose is to maintain vascular caliber. Nitric oxide may also be released by vascular cells in response to constriction. Vasoconstriction associated with CSD and/or astrocyte calcium waves could also result in an uncoupling of blood flow and metabolic activity. Reduced parenchymal blood flow in the face of intense neuronal and glial activity could cause the release of cellular metabolites and lower extracellular $\mathrm{pH}$ to trigger a nociceptive response. For example, transient receptor potential channels or acid-sensing ion channels on nociceptive trigeminal neurons could be activated under these conditions (McCleskey and Gold, 1999).

\section{THE BLOOD-BRAIN BARRIER}

In addition to changes in vascular caliber, cortical waves may be associated with changes in the permeability of the vasculature. The blood-brain barrier is a structural and functional barrier comprised of both vascular and astrocytic components. Tight junctions between endothelial cells, and astrocyte endfeet that are closely apposed to the abluminal surface of the vessel, create a specialized structure with extremely limited permeability (Persidsky et al., 2006). This structure, in combination with a variety of membrane pumps and transporters, results in a barrier that allows only highly selective entry of glucose, amino acids, and other specific molecules from the vasculature into the brain. It has long been speculated that migraine is associated with a breakdown of the blood-brain barrier, partly because of temporal differences in the efficacy of abortive medications based on the phase of the migraine attack in which they are delivered (Harper et al., 1977). There are a few case reports of cortical gadolinium enhancement on MRI in migraineurs during attacks, indicating increased permeability of the blood-brain barrier (Smith et al., 2002; Dreier et al., 2005) (although this is a rare exception to the typically normal MRI studies of most migraine patients). CSD in rats has been associated with increased permeability of the blood-brain barrier as indicated by activation and upregulation of matrix metalloproteinase-9 (Gursoy-Ozdemir et al., 2004). This study provides evidence that one of the consequences of the propagated cortical activity in migraine may be an increase in blood-brain barrier permeability, and raises the possibility that leakage across the bloodbrain barrier could be a mechanism for migraine pain. While inhibitors of plasma protein extravasation have not been effective in treating migraine, these agents were developed with models where the trigger for plasma extravasation was neurogenic inflammation, rather than spreading depression. Thus, they do not necessarily exclude a role for blood-brain barrier breakdown that is due to cortical waves.

\section{GENETIC MODULATION OF MIGRAINE MECHANISMS}

A wide variety of factors may influence the individual cellular components of migraine. As discussed above, genetic variations in the function of neurons, astrocytes, and vascular cells have each been implicated in different forms of migraine. It is important to note that mutations in genes expressed in multiple cell types and responsible for a variety of cellular 
functions all result in a similar clinical phenotype. This indicates that there are multiple points of entry to a final common pathway leading to migraine. For the FHM mutations, the resulting alterations in function are all likely to lead to an increase in cortical excitability. A knockin mouse expressing one identified mutation in the P/Q-type calcium channel associated with FHM1 provides compelling evidence for increased cortical excitability related to migraine (van den Maagdenberg et al., 2004). The threshold for evoking CSD in this mouse is significantly reduced compared with wild-type controls, and the rate of propagation of CSD is increased. For the FHM2 mutation in the astrocyte $\mathrm{Na}^{+} / \mathrm{K}^{+}$ATPase, there is as yet no mouse model expressing this mutation. However, a reduced function of this pump, as has been shown in vitro in cells expressing the FHM2 mutations, would be expected to increase cortical excitability. Consistent with this concept, the $\mathrm{Na}^{+} / \mathrm{K}^{+}$inhibitor ouabain reliably evokes CSD in rodent models (Basarsky et al., 1998). The increased excitability of neurons associated with the voltage-gated $\mathrm{Na}^{+}$channel mutations responsible for FHM3 would also be expected to increase cortical excitability. Thus, at least for FHM genes, an increased cortical excitability potentially predisposing to the occurrence of cortical waves may represent a common mechanism leading to the similar clinical phenotype. It will be interesting to determine if other migraine genes share this mechanism.

\section{SEX MODULATION OF MIGRAINE MECHANISMS}

Sex is another important modulator of migraine pathophysiology. The mechanisms underlying the dramatically greater prevalence of migraine in adult females are poorly understood. But as with genetic factors, sex may influence migraine through modulation of cortical excitability. We have found that the threshold for induction of CSD in mice is significantly reduced in female mice as compared with males (Brennan et al., 2007a). This result was obtained in randomly sampled mice without monitoring of the estrous cycle, such that the results cannot be easily explained by a reduced threshold for CSD occurring only at a single specific phase of the hormonal cycle. This increased susceptibility to CSD could involve changes in cortical excitability that are mediated by sustained exposure to gonadal hormones over days to months, developmental effects of hormones, chromosomal effects that are independent of hormones, or any combination of the above. Another potential mechanism for modulation of migraine by sex is alteration of sensitization of trigeminal nociceptive pathways. Martin et al. (2007) found that there was increased sensitization of nociceptive neuronal responses in the trigeminal nucleus caudalis in association with specific phases of the estrous cycle in rat, suggesting that alteration in the frequency and pattern of migraine associated with the menstrual cycle could involve changes in the sensitization of the trigeminal pathway.

\section{AN INTEGRATED MODEL}

The following is a hypothesized model for the sequence of events leading to migraine. In the cortex, a variety of different factors (genetic, neurochemical, ionic, and/or hormonal) lead to a dysregulation of excitability. This altered excitability triggers propagated waves of neuronal and glial activation, including astrocyte calcium waves, that are similar to, but not necessarily identical to, classical CSD. These cortical waves are associated with propagated changes in vascular caliber, and also breakdown of the blood-brain barrier. Cortical waves 
are also associated with release of a wide variety of neurotransmitters and neuromodulators, as well as changes in the ionic composition of the extracellular space that can activate nociceptive signaling pathways. These signaling pathways may also be activated by vascular constriction associated with cortical waves, through the release of messengers such as CGRP and nitric oxide that also evoke vasodilation, as well as by changes in the extracellular chemical and ionic condition resulting from vascular-metabolic uncoupling. Events occurring primarily in the cortex are then transmitted via peripheral trigeminal pathways to the brainstem, where second-order neurons are activated and eventually become sensitized. Alternatively, second-order neurons in the brainstem are activated in parallel to, or even in the absence of, cortical phenomena by changes in cellular excitability that may be similar to those described in the cortex. Third-order neurons in the thalamus and cortex are then activated and sensitized.

Specific neuronal, glial, and vascular signaling pathways may represent distinct targets for acute and preventive migraine therapies (Figure 7.2). An increased understanding of these pathways is now more accessible with advanced imaging and physiological recording techniques in combination with novel genetic models and molecular and pharmacological approaches. It is likely that there is extensive variation in the specific pathways that lead to migraine in different individuals. There may be opportunities to tailor new treatments to these specific molecular and cellular pathways in order to maximize efficacy and tolerability of therapy for this complex neurobiological disorder.

\section{References}

Adams RW, Lambert GA, Lance JW. Stimulation of brainstem nuclei in the cat: effect on neuronal activity in the primary visual cortex of relevance to cerebral blood flow and migraine. Cephalalgia. 1989; 9:107-118. [PubMed: 2743409]

Afridi SK, Matharu MS, Lee L, et al. A PET study exploring the laterality of brainstem activation in migraine using glyceryl trinitrate. Brain. 2005; 128:932-939. [PubMed: 15705611]

Ambrosini A, Shoenen J. Electrophysiological response patterns of primary sensory cortices in migraine. J Headache Pain. 2006; 7:377-388. [PubMed: 17164990]

Antal A, Arlt S, Nitsche MA, et al. Higher variability of phosphene thresholds in migraineurs than in controls: a consecutive transcranial magnetic stimulation study. Cephalalgia. 2006; 26:865-870. [PubMed: 16776703]

Aurora SK, Cao Y, Bowyer SM, et al. The occipital cortex is hyperexcitable in migraine: experimental evidence. Headache. 1999; 39:469-476. [PubMed: 11279929]

Aurora SK, Welch KMA, Al-Sayed F. The threshold for phosphenes is lower in migraine. Cephalalgia. 2003; 23:258-263. [PubMed: 12716342]

Aurora SK, Barrodale PM, Tipton RL, et al. Brainstem dysfunction in chronic migraine as evidenced by neurophysiological and positron emission tomography studies. Headache. 2007; 47:996-1003. discussion 1004-1007. [PubMed: 17635590]

Ayata C, Shin HK, Salomone S, et al. Pronounced hypoperfusion during spreading depression in mouse cortex. J Cereb Blood Flow Metab. 2004; 24:1172-1182. [PubMed: 15529018]

Bahra A, Matharu MS, Buchel C, et al. Brainstem activation specific to migraine headache. Lancet. 2001; 357:1016-1017. [PubMed: 11293599]

Basarsky TA, Duffy SN, Andrew RD, et al. Imaging spreading depression and associated intracellular calcium waves in brain slices. J Neurosci. 1998; 18:7189-7199. [PubMed: 9736642]

Boers P, Donaldson C, Zagami A, et al. Naratriptan has a selective inhibitory effect on trigeminovascular neurones at central 5-HT1A and 5-HT1B/1D receptors in the cat: implications for migraine therapy. Cephalalgia. 2004; 24:99-109. [PubMed: 14728705] 
Bolay H, Reuter U, Dunn AK, et al. Intrinsic brain activity triggers trigeminal meningeal afferents in a migraine model. Nat Med. 2002; 8:136-142. [PubMed: 11821897]

Brennan KC, Romero-Reyes MR, Lopez-Valdes HE, et al. Reduced threshold for cortical spreading depression in female mice. Ann Neurol. 2007a; 61:603-606. [PubMed: 17474110]

Brennan KC, Beltran-Parrazal L, Lopez-Valdes HE, et al. Distinct vascular conduction with cortical spreading depression. J Neurophysiol. 2007b; 97:4143-4151. [PubMed: 17329631]

Cao Y, Welch KM, Aurora S, et al. Functional MRI-BOLD of visually triggered headache in patients with migraine. Arch Neurol. 1999; 56:548-554. [PubMed: 10328249]

Cao Y, Aurora SK, Nagesh V, et al. Functional MRI-BOLD of brainstem structures during visually triggered migraine. Neurology. 2002; 59:72-78. [PubMed: 12105310]

Charles A. Intercellular calcium waves in glia. Glia. 1998; 24:39-49. [PubMed: 9700488]

Charles AC, Merrill JE, Dirksen ER, et al. Intercellular signaling in glial cells: calcium waves and oscillations in response to mechanical stimulation and glutamate. Neuron. 1991; 6:983-992. [PubMed: 1675864]

Chuquet J, Hollender L, Nimchinsky EA. High-resolution in vivo imaging of the neurovascular unit during spreading depression. J Neurosci. 2007; 27:4036-4044. [PubMed: 17428981]

Cutrer FM, Black DF. Imaging findings of migraine. Headache: J Head Face Pain. 2006; 46:10951107.

D'Hanis W, Linke R, Yilmazer-Hanke DM. Topography of thalamic and parabrachial calcitonin generelated peptide (CGRP) immunoreactive neurons projecting to subnuclei of the amygdala and extended amygdala. J Comp Neurol. 2007; 505:268-291. [PubMed: 17879271]

De Fusco M, Marconi R, Silvestri L, et al. Haploinsufficiency of ATP1A2 encoding the $\mathrm{Na}^{+} / \mathrm{K}^{+}$pump alpha2 subunit associated with familial hemiplegic migraine type 2. Nat Genet. 2003; 33:192-196. [PubMed: 12539047]

Denuelle M, Fabre N, Payoux P, et al. Hypothalamic activation in spontaneous migraine attacks. Headache. 2007; 47:1418-1426. [PubMed: 18052951]

Dichgans M, Mayer M, Uttner I, et al. The phenotypic spectrum of CADASIL: Clinical findings in 102 cases. Ann Neurol. 1998; 44:731-739. [PubMed: 9818928]

Dichgans M, Freilinger T, Eckstein G, et al. Mutation in the neuronal voltage-gated sodium channel SCN1A in familial hemiplegic migraine. Lancet. 2005; 366:371-377. [PubMed: 16054936]

Dreier JP, Jurkat-Rott K, Petzold GC, et al. Opening of the blood-brain barrier preceding cortical edema in a severe attack of FHM type II. Neurology. 2005; 64:2145-2147. [PubMed: 15985592]

Ellrich J, Messlinger K, Chiang CY, et al. Modulation of neuronal activity in the nucleus raphe magnus by the 5-HT(1)-receptor agonist naratriptan in rat. Pain. 2001; 90:227-231. [PubMed: 11207394]

Fellin T, Sul JY, D' Ascenzo M, et al. Bidirectional astrocyte-neuron communication: the many roles of glutamate and ATP. Novartis Found Symp. 2006; 276:208-217. discussion 217-221, 233-207, 275-281. [PubMed: 16805432]

Filosa JA, Bonev AD, Straub SV, et al. Local potassium signaling couples neuronal activity to vasodilation in the brain. Nat Neurosci. 2006; 9:1397-1403. [PubMed: 17013381]

Fragoso YD, Brooks JB. Two cases of lesions in brainstem in multiple sclerosis and refractory migraine. Headache. 2007; 47:852-854. [PubMed: 17578534]

Geraud G, Denuelle M, Fabre N, et al. Positron emission tomographic studies of migraine. Rev Neurol (Paris). 2005; 161:666-670. [PubMed: 16141953]

Gerwig M, Niehaus L, Kastrup O, et al. Visual cortex excitability in migraine evaluated by single and paired magnetic stimuli. Headache: J Head Face Pain. 2005; 45:1394-1399.

Goadsby PJ. Neurovascular headache and a midbrain vascular malformation: evidence for a role of the brainstem in chronic migraine. Cephalalgia. 2002; 22:107-111. [PubMed: 11972577]

Goadsby PJ, Duckworth JW. Low frequency stimulation of the locus coeruleus reduces regional cerebral blood flow in the spinalized cat. Brain Res. 1989; 476:71-77. [PubMed: 2914215]

Goadsby PJ, Edvinsson L. Human in vivo evidence for trigeminovascular activation in cluster headache. Neuropeptide changes and effects of acute attacks therapies. Brain. 1994; 117:427-434. [PubMed: 7518321] 
Goadsby PJ, Hoskin KL. Inhibition of trigeminal neurons by intravenous administration of the serotonin (5HT) 1B/D receptor agonist zolmitriptan (311C90): are brain stem sites therapeutic target in migraine? Pain. 1996; 67:355-359. [PubMed: 8951929]

Gursoy-Ozdemir Y, Qiu J, Matsuoka N, et al. Cortical spreading depression activates and upregulates MMP-9. J Clin Invest. 2004; 113:1447-1455. [PubMed: 15146242]

Guthrie PB, Knappenberger J, Segal M, et al. ATP released from astrocytes mediates glial calcium waves. J Neurosci. 1999; 19:520-528. [PubMed: 9880572]

Haas DC, Kent PF, Friedman DI. Headache caused by a single lesion of multiple sclerosis in the periaqueductal gray area. Headache. 1993; 33:452-455. [PubMed: 8262789]

Hadjikhani N, Sanchez del Rio M, Wu O, et al. Mechanisms of migraine aura revealed by functional MRI in human visual cortex. Proc Natl Acad Sci. 2001; 98:4687-4692. [PubMed: 11287655]

Harper AM, MacKenzie ET, McCulloch J, et al. Migraine and the blood-brain barrier. Lancet. 1977; 1:1034-1036. [PubMed: 67489]

Haydon PG, Carmignoto G. Astrocyte control of synaptic transmission and neurovascular coupling. Physiol Rev. 2006; 86:1009-1031. [PubMed: 16816144]

Hernandez-Caceres J, Macias-Gonzalez R, Brozek G, et al. Systemic ketamine blocks cortical spreading depression but does not delay the onset of terminal anoxic depolarization in rats. Brain Res. 1987; 437:360-364. [PubMed: 3435842]

Hoskin KL, Kaube H, Goadsby PJ. Central activation of the trigeminovascular pathway in the cat is inhibited by dihydroergotamine. A c-Fos and electrophysiological study. Brain. 1996; 119:249256. [PubMed: 8624686]

Jenkins DW, Langmead CJ, Parsons AA, et al. Regulation of calcitonin gene-related peptide release from rat trigeminal nucleus caudalis slices in vitro. Neurosci Lett. 2004; 366:241-244. [PubMed: 15288426]

Kruuse C, Thomsen LL, Birk S, et al. Migraine can be induced by sildenafil without changes in middle cerebral artery diameter. Brain. 2003; 126:241-247. [PubMed: 12477710]

Kunkler PE, Kraig RP. P/Q $\mathrm{Ca}^{2+}$ channel blockade stops spreading depression and related pyramidal neuronal $\mathrm{Ca}^{2+}$ rise in hippocampal organ culture. Hippocampus. 2004; 14:356-367. [PubMed: 15132435]

Leão AAP. Spreading depression of activity in the cerebral cortex. J Neurophysiol. 1944; 7:359-390.

Leão AAP. Further observations on the spreading depression of activity in the cerebral cortex. J Neurophysiol. 1947; 10:409-414. [PubMed: 20268874]

Levy D, Strassman AM. Mechanical response properties of A and C primary afferent neurons innervating the rat intracranial dura. J Neurophysiol. 2002; 88:3021-3031. [PubMed: 12466427]

Martin VT, Lee J, Behbehani MM. Sensitization of the trigeminal sensory system during different stages of the rat estrous cycle: implications for menstrual migraine. Headache: J Head Face Pain. 2007; 47:552-563.

Matharu MS, Goadsby PJ. Persistence of attacks of cluster headache after trigeminal nerve root section. Brain. 2002; 125:976-984. [PubMed: 11960888]

McCleskey EW, Gold MS. Ion channels of nociception. Annu Rev Physiol. 1999; 61:835-856. [PubMed: 10099712]

Mulligan SJ, MacVicar BA. Calcium transients in astrocyte endfeet cause cerebrovascular constrictions. Nature. 2004; 431:195-199. [PubMed: 15356633]

Offenhauser N, Zinck T, Hoffmann J, et al. CGRP release and cfos expression within trigeminal nucleus caudalis of the rat following glyceryltrinitrate infusion. Cephalalgia. 2005; 25:225-236. [PubMed: 15689199]

Olesen J, Friberg L, Olsen TS, et al. Timing and topography of cerebral blood flow, aura, and headache during migraine attacks. Ann Neurol. 1990; 28:791-798. [PubMed: 2285266]

Ophoff RA, Terwindt GM, Vergouwe MN, et al. Familial hemiplegic migraine and episodic ataxia type-2 are caused by mutations in the $\mathrm{Ca}^{2+}$ channel gene CACNL1A4. Cell. 1996; 87:543-552. [PubMed: 8898206] 
Peeters M, Gunthorpe MJ, Strijbos PJ, et al. Effects of pan- and subtype-selective NMDA receptor antagonists on cortical spreading depression in the rat: therapeutic potential for migraine. $\mathrm{J}$ Pharmacol Exp Ther. 2007; 321:564-572. [PubMed: 17267580]

Penfield W. A contribution to the mechanism of intracranial pain. Assoc Res Nerv Ment Dis. 1935; 15:399-416.

Persidsky Y, Ramirez S, Haorah J, et al. Blood-brain Barrier: structural components and function under physiologic and pathologic conditions. J Neuroimmune Pharmacol. 2006; 1:223-236. [PubMed: 18040800]

Peters O, Schipke CG, Hashimoto Y, et al. Different mechanisms promote astrocyte $\mathrm{Ca}^{2+}$ waves and spreading depression in the mouse neocortex. J Neurosci. 2003; 23:9888-9896. [PubMed: 14586018]

Raskin NH, Hosobuchi Y, Lamb S. Headache may arise from perturbation of brain. Headache. 1987; 27:416-420. [PubMed: 3667258]

Ray BS, Wolff HG. Experimental studies in headache: Pain-sensitive structures of the head and their structural significance. Arch Surg. 1940; 41:813-856.

Richards A, van den Maagdenberg AMJM, Jen JC, et al. C-terminal truncations in human 3[prime]-5 [prime] DNA exonuclease TREX1 cause autosomal dominant retinal vasculopathy with cerebral leukodystrophy. Nat Genet. 2007; 39:1068-1070. [PubMed: 17660820]

Schoenen J. Neurophysiological features of the migrainous brain. Neurol Sci. 2006; 27(Suppl 2):S77S81. [PubMed: 16688634]

Schoonman GG, van der Grond J, Kortman C, et al. Migraine headache is not associated with cerebral or meningeal vasodilation - a 3T magnetic resonance angiography study. Brain. 2008; 131:21922200. [PubMed: 18502781]

Segall L, Mezzetti A, Scanzano R, et al. Alterations in the alpha2 isoform of Na,K-ATPase associated with familial hemiplegic migraine type 2. Proc Natl Acad Sci U S A. 2005; 102:11106-11111. [PubMed: 16037212]

Shields KG, Goadsby PJ. Serotonin receptors modulate trigeminovascular responses in ventroposteromedial nucleus of thalamus: A migraine target? Neurobiol Dis. 2006; 23:491-501. [PubMed: 16875831]

Smith M, Cros D, Sheen V. Hyperperfusion with vasogenic leakage by fMRI in migraine with prolonged aura. Neurology. 2002; 58:1308-1310.

Stankewitz A, May A. Cortical excitability and migraine. Cephalalgia. 2007; 27:1454-1456. [PubMed: 18034689]

Strassman AM, Levy D. Response properties of dural nociceptors in relation to headache. $\mathbf{J}$ Neurophysiol. 2006; 95:1298-1306. [PubMed: 16492942]

Strassman AM, Raymond SA, Burstein R. Sensitization of meningeal sensory neurons and the origin of headaches. Nature. 1996; 384:560-564. [PubMed: 8955268]

Tajti J, Uddman R, Edvinsson L. Neuropeptide localization in the 'migraine generator' region of the human brainstem. Cephalalgia. 2001; 21:96-101. [PubMed: 11422090]

Takano T, Tian GF, Peng W, et al. Astrocyte-mediated control of cerebral blood flow. Nat Neurosci. 2006; 9:260-267. [PubMed: 16388306]

van den Maagdenberg AMJM, Pietrobon D, Pizzorusso T, et al. A Cacna1a knockin migraine mouse model with increased susceptibility to cortical spreading depression. Neuron. 2004; 41:701-710. [PubMed: 15003170]

Vanmolkot KRJ, Kors EE, Turk U, et al. Two de novo mutations in the Na,K-ATPase gene ATP1A2 associated with pure familial hemiplegic migraine. Eur J Human Genetics. 2006; 14:555-560. [PubMed: 16538223]

Weiller C, May A, Limmroth V, et al. Brain stem activation in spontaneous human migraine attacks. Nat Med. 1995; 1:658-660. [PubMed: 7585147]

Welch KM, Nagesh V, Aurora SK, et al. Periaqueductal gray matter dysfunction in migraine: cause or the burden of illness? Headache. 2001; 41:629-637. [PubMed: 11554950]

Woods RP, Iacoboni M, Mazziotta JC. Bilateral spreading cerebral hypoperfusion during spontaneous migraine headache. N Engl J Med. 1994; 331:1689-1692. [PubMed: 7969360] 
Zonta M, Angulo MC, Gobbo S, et al. Neuron-to-astrocyte signaling is central to the dynamic control of brain microcirculation. Nat Neurosci. 2003; 6:43-50. [PubMed: 12469126] 


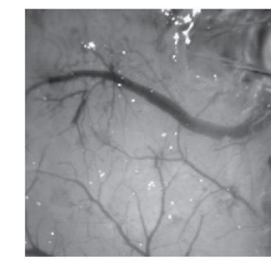

A

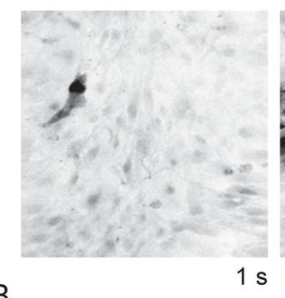

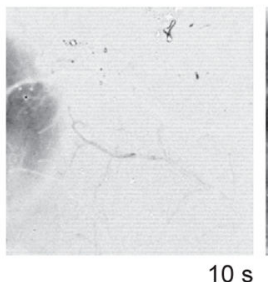

$10 \mathrm{~s}$

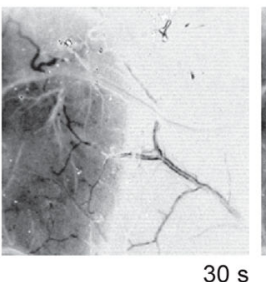

$30 \mathrm{~s}$

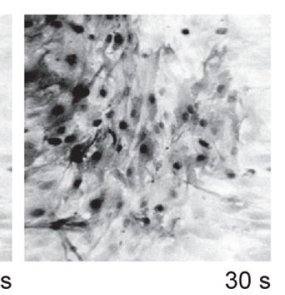

Fig. 7.1.

Cortical spreading depression (CSD) and astrocyte calcium waves. (A) Optical imaging of the cortical surface in a mouse. Far left panel shows image of mouse cortex visualized through the thinned skull of an anesthetized mouse (a recording electrode is seen in the upper right of the field; image scale $=1200 \times 1200 \mu \mathrm{m}$ ). Subsequent images show change in reflectance of this area of cortex over time associated with a CSD wave. A change in optical signal of the parenchyma spreads slowly across the cortex. Dilation (darkening) of surface arteries propagates ahead of the CSD wavefront, followed by constriction of these vessels accompanying the CSD wave. (B) Astrocyte calcium wave. Images show fluorescence of the calcium indicator fluo-4 with an inverse gray scale (darker gray indicates higher intracellular calcium; image scale $=400 \times 400 \mu \mathrm{m}$ ). Mechanical stimulation of a single cell with a micropipette evokes a wave of increased calcium concentration that spreads from cell to cell over as many as hundreds of cells. The temporal and spatial characteristics of this wave are remarkably similar to those of CSD. 


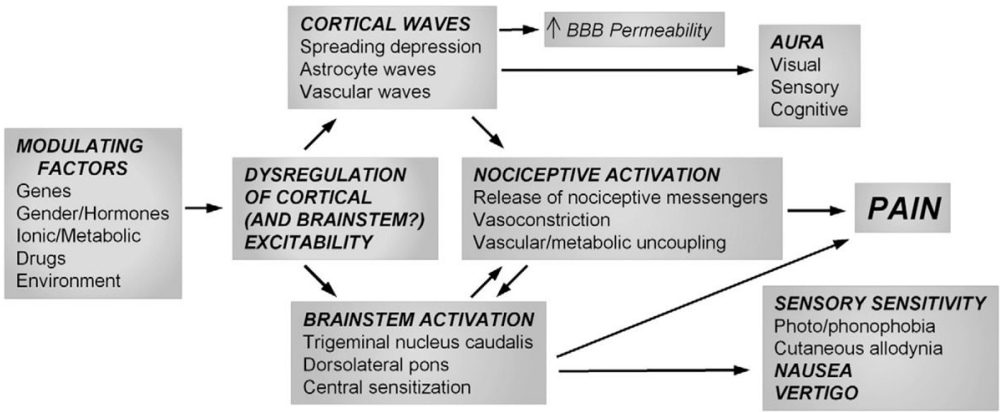

Fig. 7.2.

Schematic of hypothesized sequence of events in migraine. Each step in the sequence may have discrete thresholds, and involve distinct molecular, cellular, and neurochemical pathways. This complexity may underlie the heterogeneous and variable clinical presentation of migraine. BBB, blood-brain barrier. 